\title{
Adaptive Power Control Algorithm with Stabilization Zone for Third Generation Mobile Networks
}

\author{
Jad Nasreddine, Loutfi Nuaymi and Xavier Lagrange \\ GET / ENST Bretagne \\ e-mail\{jad.nasreddine, loutfi.nuaymi, xavier.lagrange\}@enst-bretagne.fr
}

\begin{abstract}
In this paper, we propose an evolution of adaptive power control algorithms for Universal Mobile Telecommunications System (UMTS). The proposed mechanism uses an adaptive power step to carry out with high fluctuations in the radio interface. Furthermore, we propose the integration of a stabilization zone where the transmitted power is kept constant to reduce oscillations around the target QoS level. In this algorithm, only the interpretation of power control feedback commands is ameliorated by adding some intelligence to mobiles and base stations. Moreover, the standardized radio interface protocol is used without modification. The algorithm can be used in both link directions of the Frequency Division Duplex (FDD) mode and in the downlink of the Time Division Duplex (TDD) mode, where closed-loop power control algorithms are used. Simulation results show that the proposed algorithm decreases the outage probability and reduces drastically the transmitted power, which are the main objectives of power control algorithms.

Index Terms- Adaptive power control, Stabilization zone, UMTS, TDD, FDD.
\end{abstract}

\section{Introduction}

Mobile network radio interface is a very challenging environment due to interference and unstable radio propagation characteristics. Power control process is one of the most efficient tools to assess this problem. Power control algorithms in the Universal Mobile Telecommunications Systems (UMTS) were specified in the $3^{\text {rd }}$ Generation Partnership Project (3GPP) standardization forum [1][2] for FDD and TDD modes respectively. Proposals for UMTS involve a combination of inner-loop and outer-loop power control algorithms.

The main objective of the outer-loop algorithm, performed by mobile and RNC equipments, is to dynamically adjust the target Carrier-to-Interference Ratio (CIR), called $\mathrm{CIR}_{\text {target }}$, according to a fixed Block-Error Rate (BLER) or Bit-Error Rate (BER) and depending on propagation channel characteristics. The $\mathrm{CIR}_{\text {target }}$ of a given radio channel is achieved by the inner-loop power control algorithm, which is performed by both mobiles and Node Bs [3].

In the downlink of the TDD mode and in both link directions of the FDD mode, inner-loop power control algorithms are based on a closed-loop control. In uplink of the TDD mode, an open-loop control is used: the closed loop power control is used mainly to assess the problem of fast fading that have high impact on the downlink of the TDD mode, where the number of transmitter is not very high. In the uplink however, the total interference slightly changes 
with fast channel fading due to the big number of transmitters. Moreover, the TDD mode is characterized by a channel reciprocity that can be used to perform open-loop power control algorithms [4]. The closed-loop power control algorithm is used after an initial phase that uses an open-loop power control algorithm [1]. The drawback of the power control variant in the TDD mode is that the rate of the feedback command in slow rate applications is reduced to $100 \mathrm{~Hz}$ compared to $1500 \mathrm{~Hz}$ in the FDD mode [5].

The transmission unit in both FDD and TDD modes is a $10 \mathrm{~ms}$ frame. Each frame is divided into 15 time slots. Two algorithms were proposed in the 3GPP specification [1] for FDD. In the first algorithm, the transmitted power is updated each time slot while it may be updated once each 5 time slots in the second algorithm. Only the first algorithm is studied in this paper. Every time slot, the mobile estimates the received $C I R$ and compares it to $C I R_{\text {target }}$. When the estimated $C I R$, denoted $C I R_{\text {est }}$, is higher than $C I R_{\text {target }}$, the Transmitted Power Command (TPC) is set to " 0 " and the transmitted power is decreased by $\Delta_{\text {step }} \mathrm{dB}$. Otherwise, the TPC is set to " $1 "$ and the transmitted power is increased by $\Delta_{\text {step }} \mathrm{dB}$. In the TDD mode, the transmitted power can be updated each frame by use of the same procedure as in the FDD mode [2]. In most UMTS formats, the TPC command is repeated on two-bits as an errorprotection redundancy. The power control step is fixed by higher layers and it depends on radio and service parameters. Several values of the order of $1 \mathrm{~dB}$ are proposed in the specification [1]. In figure 1, we show the downlink closed-loop power control process.

In this paper, an evolution of adaptive closed-loop power control is proposed by adding a stabilization zone above the $C I R_{\text {target }}$ and ameliorating feedback commands interpretation. In Section II, high fluctuations in the radio interface and oscillation problems are presented. Moreover, possible solutions of each of these problems are investigated. The proposed algorithm and its implementation are described in Section III. System model is described in Section IV and results are discussed in Section V. Conclusions and useful comments are highlighted in the last section.

\section{Problem Formulation}

\subsection{Adaptive steps}

In wireless telecommunications systems, frequent fluctuations occur in the radio interface. Therefore, the received CIR may change significantly and very fast. In many cases, the presently-proposed 3GPP power control may not be able to carry out with these variations because the power update step is fixed. In other words, the power is updated with a fixed step whatever the magnitude of variations in pathloss and interference profile. This leads to the degradation of QoS levels and some channels may be dropped. In figure 2, the CIR of a mobile is dropped to a low level suddenly (e.g. a big obstacle appears between the mobile and its
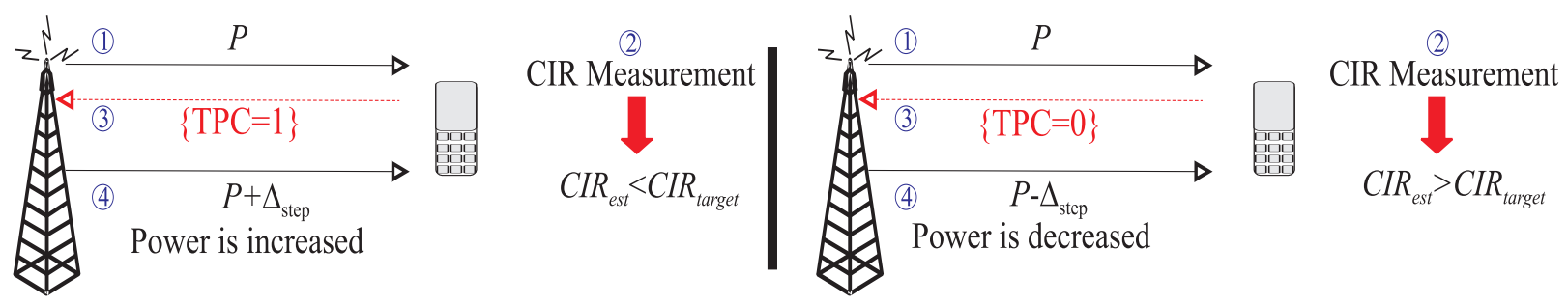

Figure 1: 3GPP Downlink inner-loop power control algorithm steps. 


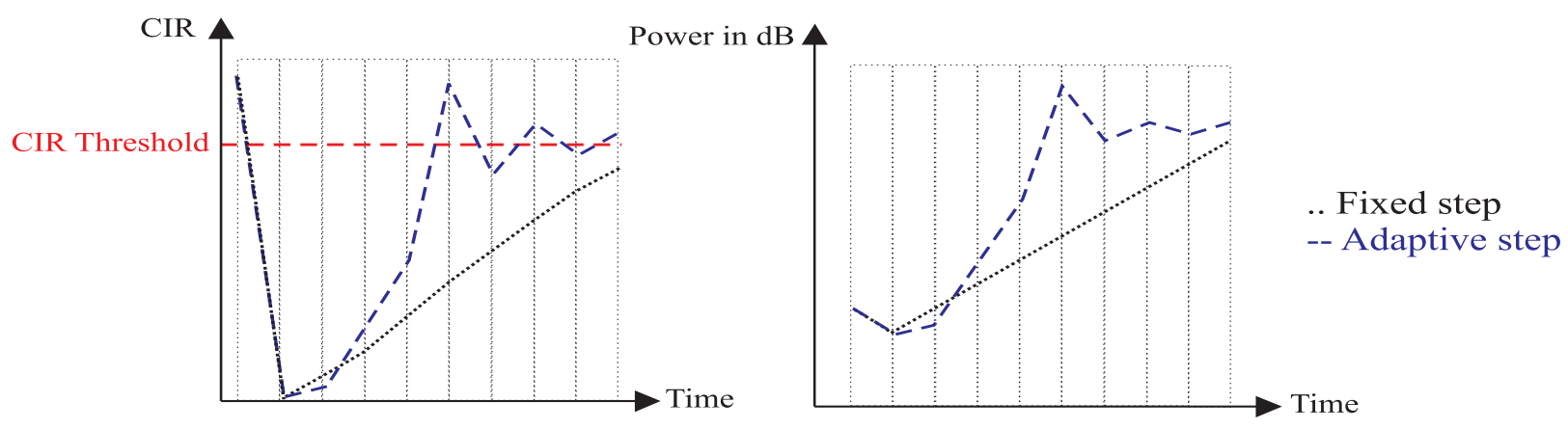

Figure 2: A comparison between fixed-step and adaptive-step power control convergence.

server). For small $\Delta_{\text {step }}$, the convergence of the fixed-step power control is very slow. However, if high value of $\Delta_{\text {step }}$ is used always, the oscillation around the $\mathrm{CIR}_{\text {target }}$ will have high variance. In order to avoid this type of situation, adaptive-step power control may be used. In this type of power control, the power-update step accommodates CIR fluctuations. Some methods were proposed to increase the adaptation of power control algorithms by using asymmetric power control steps: different step values for increasing and decreasing power [5]. Other methods suggest an adaptive power variation step in order to decrease oscillations around $\mathrm{CIR}_{\text {target }}$ and to increase the speed of the power control when the difference between $\mathrm{CIR}_{\text {est }}$ and $\mathrm{CIR}_{\text {target }}$ is very high [6][7]. In the Adaptive-Step Power Control (ASPC) algorithm proposed in [6], if the base station detects the same TPC from a mobile in a set of consecutive slots, the step dedicated to this mobile is increased. On the contrary, if an alternative sequence of up and down TPCs of a mobile are received by a base station, the step dedicated to this mobile is reduced. The authors of [8][9][10] propose a self-tuning predictor algorithm where the TPC is coded on 2, 3 or 4 bits instead of one. In these latter papers, the parameters of the power control change according to the quality measurements of the system level, which can be deduced from command and CIR error history. The self-tuning predictor algorithm ameliorates significantly system performance, but imposes some modifications on the radio interface protocol which is standardized by $3 \mathrm{GPP}$ bodies.

\subsection{CIR margin}

Adaptive power control algorithms reduce oscillations around the $\mathrm{CIR}_{\text {target }}$. However, the limited amplifier precision prevents transmitters from using very low power control steps; therefore, oscillations are not totally eliminated. CIR oscillations appear due to the fact that the power is either increased or decreased even if the CIR is very close to $\mathrm{CIR}_{\text {target }}$. Therefore, the received CIR may oscillate around $\mathrm{CIR}_{\text {target }}$ with high variance. One solution to the oscillation problem is to use a CIR margin $M$ and to define $\mathrm{CIR}_{\text {target }}+M$ as the new CIR target. The new CIR target is called $\mathrm{CIR}_{\text {target }}^{M}$ :

$$
\mathrm{CIR}_{\text {target }}^{M}=\mathrm{CIR}_{\text {target }}+M
$$

When the estimated CIR of a mobile is close to $\operatorname{CIR}_{\text {target }}^{M}$, a power decrease may degrade the CIR of the mobile in the next slot if the margin is not high enough. Therefore, a high value of $M$ is suited to minimize the outage probability. However, increasing $M$ decreases the system maximum capacity due to the high value of $\mathrm{CIR}_{\text {target }}^{M}$. Moreover, the power consumption is an increasing function of $\mathrm{CIR}_{\text {target }}^{M}$. Hence, a trade-off must be taken between the maximum capacity, power consumption and the outage probability. 

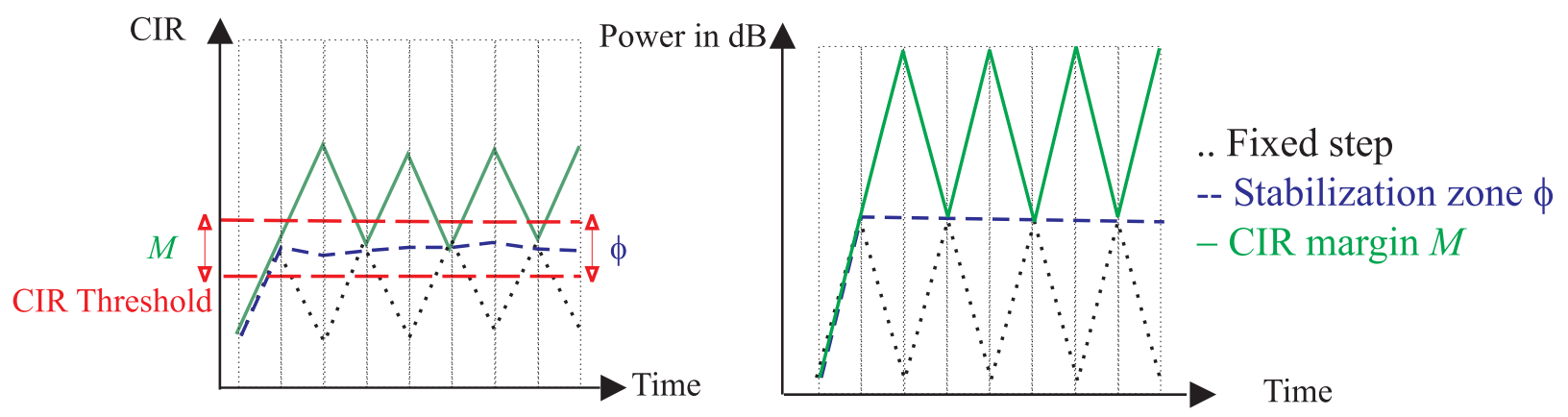

Figure 3: Solving oscillation problems by using either a CIR margin or a stabilization zone.

\subsection{Stabilization Zone}

In order to alleviate the latter problem, we proposed an adaptive-step power control with stabilization zone $\Phi[11$ ] (figure 3). In the stabilization zone, the transmitted power is kept stable by a specific algorithm. Therefore, the CIR may be kept at a quasi-fixed level above the $\mathrm{CIR}_{\text {target }}$ and the stabilization zone can be therefore considered as an adaptive CIR margin.

\section{Modified Adaptive Power Control}

In existing power control algorithms, a mobile can request only a power decrease or increase from its server. However, the mobile cannot inform its server that $\mathrm{CIR}_{\text {est }}$ is very close to $\mathrm{CIR}_{\text {target }}$ and that the power must be stabilized. Thus, $\mathrm{CIR}_{\text {est }}$ can oscillate even if the radio interface parameters are stable. Our proposed algorithm adds intelligence to the receiver in order to ask the transmitter to stabilize its power. Unlike the algorithms of [7][8][9][10], the proposed algorithm does not need more TPC bits than the conventional UMTS power control algorithm. Nevertheless, we show by simulation that the use of a stabilization zone decreases, at the same time, the outage probability and power consumption. Moreover, the tuned parameters of the adaptive schemes offer to the system a degree of flexibility in order to accommodate different mobility types (i.e. channel types).

\subsection{The Modified Adaptive Power Control Algorithm}

We propose the Modified Adaptive Power Control (MAPC) algorithm as an evolution of the existent adaptive power control algorithms. The aim of the MAPC algorithm is to reduce the oscillation variance around the $\mathrm{CIR}_{\text {target }}$, to cope with channel high fluctuations and to reduce power consumption by creating a stabilization zone $\Phi$ above the $\mathrm{CIR}_{\text {target }}$. The MAPC algorithm combines the principle of adaptive-step power control algorithms with the new concept of stabilization zone to completely eliminate oscillations. In the following, downlink power control algorithm is described. For uplink algorithm, only mobile and base station tasks should be swapped.

The MAPC algorithm uses an adaptive power control step to mitigate the impact of the radio interface variation. Moreover, when $\mathrm{CIR}_{e s t}$ of a mobile is in the stabilization zone, the mobile generates an alternative TPC sequence (up and down). Consequently, the base station reduces the power step dedicated to this mobile and stabilizes its transmitted power. The use of a stabilization zone decreases the needed margin value, which can be omitted also; therefore, the system maximum capacity is increased and the power consumption is reduced. 


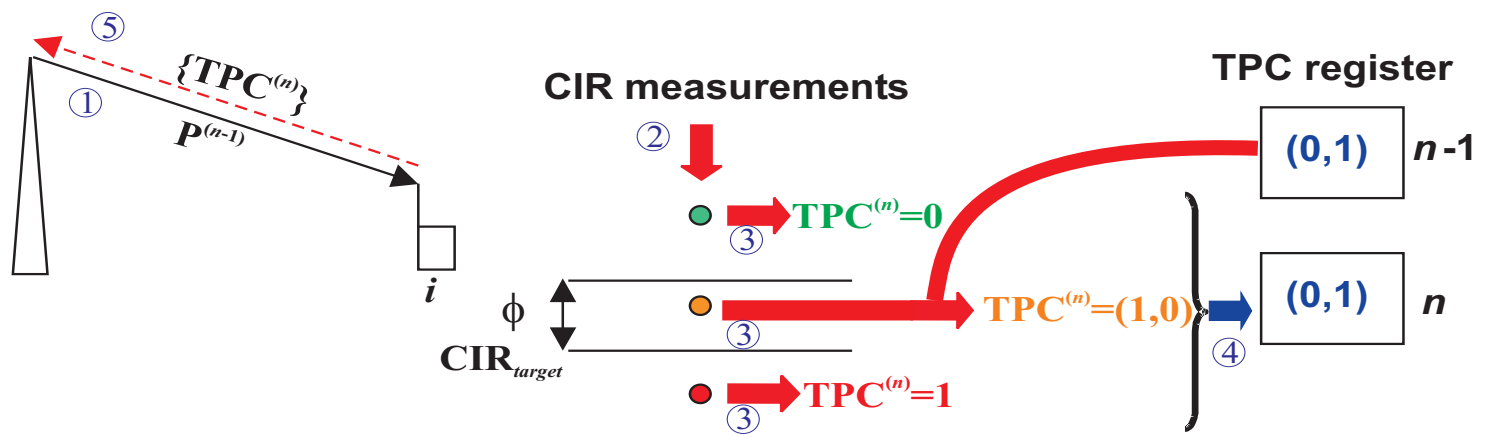

Figure 4: MAPC algorithm steps in mobiles (the TPC register can be set to 1 or 0 . The inversion of this bit is represented by $(0,1)$ at time $n-1$ and $(1,0)$ at time $n)$.

\subsection{Mobile Station Algorithm}

In the MAPC algorithm, each mobile station compares $\mathrm{CIR}_{\text {est }}$ with $\mathrm{CIR}_{\text {target }}$ and generates TPC commands according to the following algorithm (figure 4):

$\diamond$ if $\mathrm{CIR}_{\text {est }}>\mathrm{CIR}_{\text {target }}+\Phi$, then the transmitted TPC is set to "0" requesting a transmit power decrease.

$\diamond$ if $\mathrm{CIR}_{\text {target }} \leq \mathrm{CIR}_{\text {est }} \leq \mathrm{CIR}_{\text {target }}+\Phi$, then the transmitted TPC is the complementary of the previous TPC (saved in the command register) requesting an unchanged transmitted power. In this interval, mobiles generate alternative up and down TPC commands.

$\diamond$ if $\mathrm{CIR}_{\text {est }}<\mathrm{CIR}_{\text {target }}$, then the transmitted TPC is set to "1" requesting a transmit power increase.

$\diamond$ The TPC is saved in a command register of one bit.

Parameter $\Phi$ (in $\mathrm{dB}$ ) is the range of the stabilization zone. For each mobile, RRC layer in the core network sets $\mathrm{CIR}_{\text {target }}$ and $\Phi$ based on the system load, the service type and radio interface characteristics. These parameters are sent to mobile stations via signalization channels.

\subsection{Base Station Algorithm}

We remind that the $\mathrm{CIR}_{\text {target }}$ is fixed by outer-loop power control algorithm. The inner-loop of the MAPC algorithm has to be performed by the base station in the network side to increase the algorithm speed. The base station receives TPC commands from mobile stations and analyzes the command of each mobile station independently. After each power control iteration, these commands are saved in a command register, where only one bit is dedicated to each mobile.

Each base station performs the following algorithm to set the power of a mobile (figure 5):

$\diamond$ if the instantaneous and the saved TPC commands are the same, the transmitted power is updated using the previous $\Delta_{\text {step }}$, which is then multiplied by $\mu$. This schedule (i.e. updating the step after the power update) has shown better performance than the algorithm where the power is updated using the new $\Delta_{\text {step }}$ by decreasing the oscillation outside the stabilization zone. The performance results are not shown in this paper for simplicity.

$\diamond$ if the instantaneous TPC command is different from the saved command, $\Delta_{\text {step }}$ is divided by $\lambda$ and the transmitted power is maintained stable. 


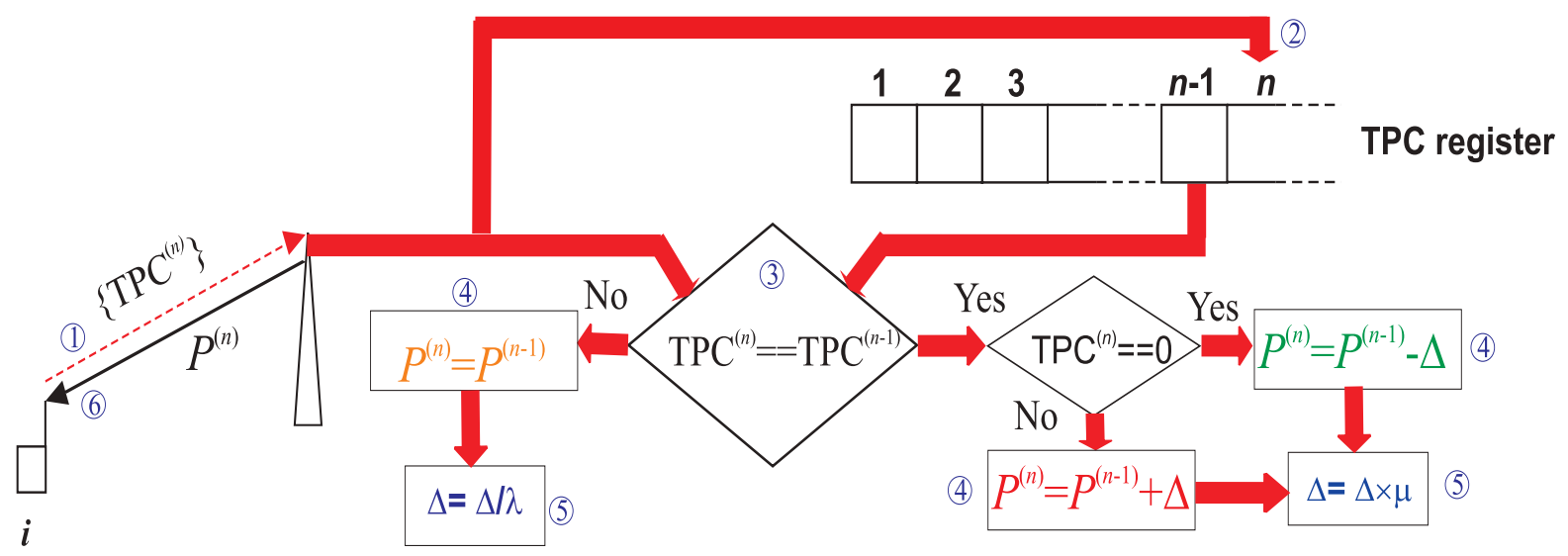

Figure 5: MAPC algorithm steps in base stations (all equations are in $\mathrm{dB}$ ).

$\diamond$ the TPC command is saved in the command register and the power control step is saved in a 3-bit register (i.e. only 8 values of power control steps are allowed).

Parameters $\lambda, \mu$ and the initial value of $\Delta_{\text {step }}$, are constant values and are fixed by the RRC layer in the core network. It should be noted that these parameters can be different for different mobiles depending on their characteristics (i.e. speed, delay sensitivity, BER sensitivity, etc.) Furthermore, the power control step margin is limited by the precision of the used amplifier and is always in the interval $\left[\Delta_{\min } ; \Delta_{\max }\right]$ where only $\Delta_{n}$ values are allowed. The value of $\Delta_{n}$ must be lower than 8 to fit with the register size.

\section{System model}

The MAPC algorithm is evaluated by simulation using Matlab. An outdoor Manhattan scenario with $5 \times 5$ blocks is used to evaluate power control algorithms [11]. The block size is $200 \times 200$ $\mathrm{m}^{2}$. Mobiles are uniformly distributed and each mobile is served by the best-received base station. A wrap-around technique is used in order to overcome cell boundary effects. Common channels, call dropping, error detection and correction, and TPC errors are not considered in simulations.

The assumed propagation model is an Okumura-Hata-Cost231 model with shadowing and fast fading; the path gain between mobile $i$ and base station $j$ at time $t$ is modeled as $G_{i, j, t}=a_{i, j} f_{t, i, j} / d_{i, j}^{3.5}$, where $d_{i, j}$ is the mobile-to-base distance expressed in meters, the coefficient $a_{i, j}$ models the shadowing effect and $f_{t, i, j}$ models the fast fading at time $t$. The shadow fading coefficient $a_{i, j}$ is a log-normal time-constant random variable with zero mean and $10 \mathrm{~dB}$ variance [12]. A correlation coefficient of 0.5 is considered between the shadow fading coefficients of a mobile and neighboring base stations. When mobility is considered, an exponential autocorrelation function $R(\Delta x)$ is used with a decorrelation distance of $5 \mathrm{~m}$ for microcells and $20 \mathrm{~m}$ for macrocells:

$$
R(\Delta x)=e^{-\frac{\Delta x}{d_{c o r}} \ln 2},
$$

where $\Delta x$ is the path length and $d_{c o r}$ is the decorrelation distance [13].

The fast fading coefficient $f_{t, i, j}$ is computed using Jakes model [14] and the method described in [15] for typical urban channel model: for each mobile $i$ at time $t$, the coefficient $f_{t, i, j}$ is computed using the following equation: 


$$
f_{t, i, j}=\left(\sum_{j=1}^{L} A_{t, i, j}^{2}\right) / \bar{f}
$$

where $\left.A_{t, i, j}\right|_{l=1 \ldots L}$ are the amplitudes of the detectable taps, $L$ is the number of these taps and $\bar{f}$ is the average value of $f$ which is used to obtain a normalized values of fading coefficients. $A_{t, i, j}$ is updated each slot using the following equation:

$$
A_{t, i, j}=\left|\sum_{l=1}^{L_{j}} T_{l, i, j} e^{2 \pi \iota f_{D, i} \cos \left(\frac{2 \pi l}{L_{j}}\right) t+\iota \theta_{l, i, j}}\right|
$$

where $\iota$ is the imaginary unit equal to the square root of $-1, L_{j}$ is the number of taps that cannot be detected and represented by tap $j[15], T_{l, i, j}$ is the amplitude of tap $l, \theta_{l, i, j}$ is the angle of arrival of tap $l$ and is uniformly distributed between $[0,2 \pi]$ and $f_{D, i}$ is the maximum Doppler shift given by:

$$
f_{D, i}=\frac{s_{i}}{\lambda}
$$

where $s_{i}$ is the speed of mobile $i$ and $\lambda$ is the wavelength corresponding to the used frequency $(2 \mathrm{GHz})$. It should be noted that the module $T_{l, i, j}$ is updated with a frequency of $2 f_{D, i}$ using a Rayleigh distribution. The averages of tap modules are given in [15] for typical urban channels and the frequency $2 f_{D, i}$ corresponds to the frequency of notches. In figure 6 , we show an example of a fast fading factor evolution of mobile moving with a speed of $50 \mathrm{Km} / \mathrm{s}$. We use this complicated model in order to guarantee some time-correlation for each fading factor.

The simulated service is $8 \mathrm{kbps}$ circuit-switched service with $100 \%$ activity factor. The $C I R_{\text {target }}$ for all mobiles is $w_{0} \mathrm{~dB}$ and a perfect $C I R$ estimation is considered. The values of $w_{0}$ are respectively $-17,-20$ and $-8 \mathrm{~dB}$ for FDD downlink, FDD uplink and TDD downlink. Orthogonality factors $\alpha$ in the downlink of the FDD mode are 0.4 and 0.06 for macrocells and microcells respectively. Moreover, the value of this factor is 0.1 in the downlink of the TDD mode, where a joint detection technique is used [16]. The factor $\alpha$ is used in the CIR equation to represent the reduction of downlink intracell interference due to the orthogonality. Hence, if $P_{\text {intra, } i}$ is the total intracell interference received by a mobile $i$, then the residual intracell interference after despreading is given by:

$$
I_{\text {Intra }, i}=\alpha P_{\text {intra }, i}
$$

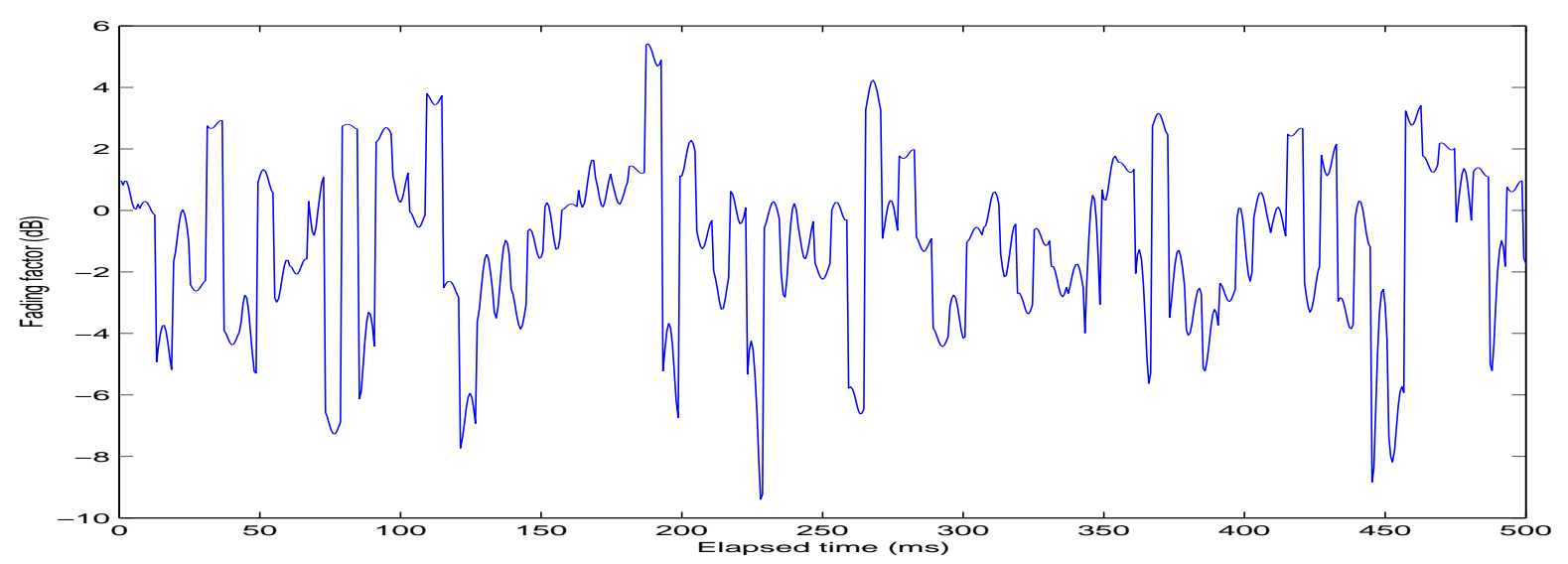

Figure 6: Simulated fast fading envelope at $2000 \mathrm{MHz}$ and receiver speed of $50 \mathrm{Km} / \mathrm{h}$. 
Table I: Power limits and receiver thermal noise.

\begin{tabular}{|l|c|c|}
\hline \multicolumn{1}{|c|}{ Parameters } & Uplink & Downlink \\
\hline Maximum BTS power (microcell) [dBm] & - & 33 \\
\hline Maximum BTS power (macrocell) [dBm] & - & 43 \\
\hline Maximum channel power [dBm] & 21 & 20 \\
\hline Power cntrl range for a BTS [dB] & - & 30 \\
\hline Power cntrl range for a channel [dB] & 65 & 25 \\
\hline Receiver thermal noise [dBm] & -103 & -98 \\
\hline
\end{tabular}

Table II: Power control parameters.

\begin{tabular}{|l|c|c|c|c|c|c|}
\cline { 2 - 7 } \multicolumn{1}{c|}{} & $\Delta_{\text {step }}$ & $\lambda$ & $\mu$ & $\Delta_{\max }$ & $\Delta_{\min }$ & $\Phi$ \\
\hline pedestrian and fixed users & $1 \mathrm{~dB}$ & 2 & 4 & $4 \mathrm{~dB}$ & $0.25 \mathrm{~dB}$ & $0.3 \mathrm{~dB}$ \\
\hline Vehicular users & $1 \mathrm{~dB}$ & 2 & 2 & $2 \mathrm{~dB}$ & $1 \mathrm{~dB}$ & $0.7 \mathrm{~dB}$ \\
\hline
\end{tabular}

Class 3 mobile stations are used: the transmitted power limits and the receiver thermal noise are presented in table I [17]. The initial transmitted powers in Monte Carlo simulations have random values between the minimum value and $10 \%$ of the maximum transmitted power, whereas an open-loop scheme is used to compute these power in the dynamic simulations. The adaptive power control parameters are presented in table II.

Algorithm performance is evaluated using frame-outage probability, which is the probability that the geometric average of the received $C I R$ over one Transmission Time Interval (TTI, e.g. $20 \mathrm{~ms}$ ) falls below $C I R_{\text {target }}$. We use the frame-outage probability instead of the instantaneous outage probability because the block error rate (BLER) is generally measured each TTI and the relation between the BLER and the CIR is approximately linear in the logarithmic scale. This scheme leads to a more accurate performance evaluation especially if the $C I R$ is in continuous fluctuations [18].

\section{Simulations and Results}

First, we evaluate the system performance by Monte Carlo simulations for 200 independent mobile configurations. In each configuration, 600 iterations are simulated (i.e. 4 seconds in the FDD mode and 1 minute in the TDD mode) with the $3 \mathrm{GPP}$ variant algorithm (with a CIR margin), the ASPC algorithm and the MAPC algorithm. The limited simulation time does not affect the mean frame-outage probability nor the mean total transmitted power due to the fast convergence of the three algorithms (i.e. less than 50 iterations). After the convergence, the system holds on in a periodic state, where the same performance is repeated at a fixed period. We remind that the ASPC does not use a stabilization zone. Moreover, the MAPC algorithm ameliorates the adaptive scheme of the ASPC algorithm in addition to the proposition of the stabilization zone. We emphasize here that table II shows the adaptive power control parameters that optimize the performance of the adaptive algorithms.

We assume that mobiles are active in the downlink during all simulation time. The mean transmitted power and the mean frame-outage probability are averaged over the simulation period.

We then analyze the performance of the 3GPP variant, the MAPC and the ASPC algorithms in a discrete-time simulation with mobility and a mean call duration of $120 \mathrm{~s}$. The 


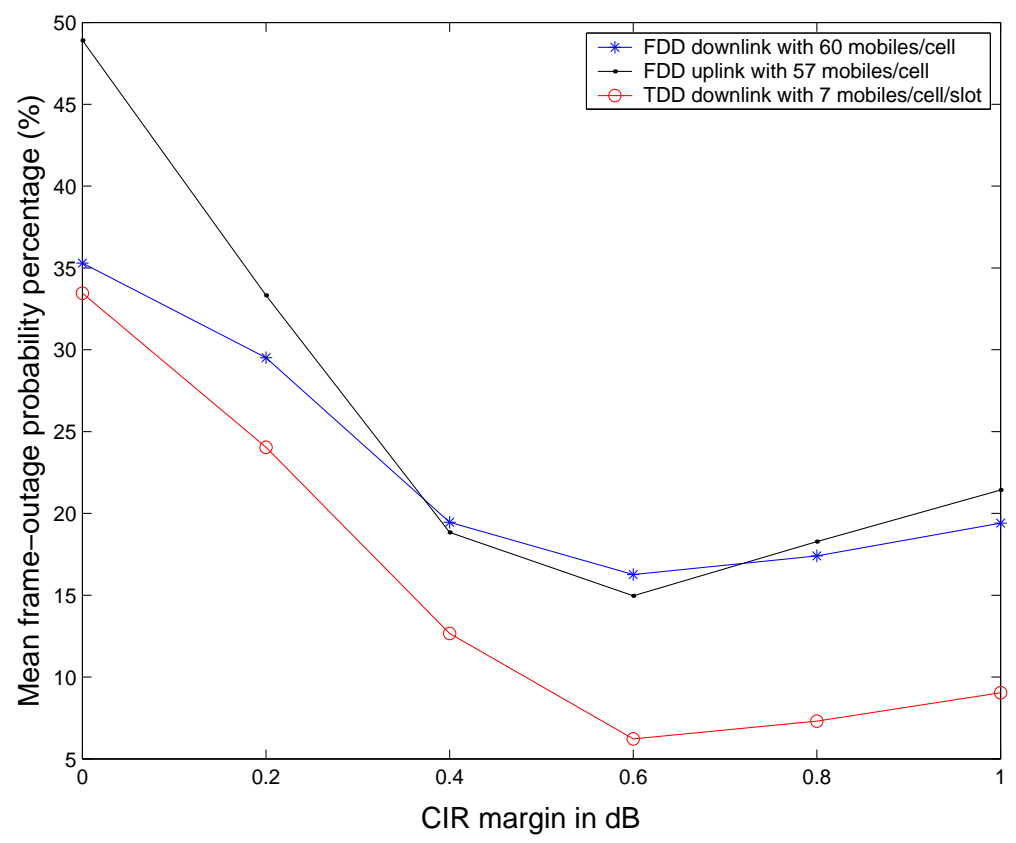

Figure 7: The mean frame-outage probability percentage for different values of the margin $M$ when the 3GPP power control algorithm is used.

simulation reflects the behavior of the system during 10 minutes (i.e. 900000 time slots). Mobile speed is considered as constant for each mobile during its presence in the system, and it has a uniform distribution for all mobiles. Two sets of simulations where performed: simulation with pedestrian users and simulation with vehicular users. In the first set, mobile speed has $3 \mathrm{~km} / \mathrm{h}$ average and $0.3 \mathrm{~km} / \mathrm{h}$ deviation whereas in the second set mobile speed has $50 \mathrm{~km} / \mathrm{h}$ average and $3 \mathrm{~km} / \mathrm{h}$ deviation. Moreover, we consider microcells with a block size of $0.2 \mathrm{x} 0.2$ $\mathrm{km}^{2}$ for the first set and macrocells with a block size of $1 \mathrm{x} 1 \mathrm{Km}^{2}$ for the second. The terminal position is updated when the terminal moves $5 \mathrm{~m}$ and only hard-handover is considered with 3 $\mathrm{dB}$ margin [12].

\subsection{CIR Margin Impact}

In figure 7 , we present the impact of CIR margin (without stabilization zone) on the mean frame-outage probability when the $3 \mathrm{GPP}$ power control algorithm is used for downlink and uplink in the FDD mode and for downlink in the TDD mode. Cell loads are respectively 60, 57 and 7 mobiles/cell/slot for downlink FDD, uplink FDD and downlink TDD. The minimum mean frame-outage probability is reached with a $0.6 \mathrm{~dB}$ CIR margin in the three systems, and therefore, system maximum capacity is reached with a $0.6 \mathrm{~dB}$ CIR margin. The mean frameoutage percentage is drastically reduced when the optimal CIR margin is used (e.g. from $34 \%$ without CIR margin to $6 \%$ with the optimal CIR margin in the TDD mode). Moreover, we studied the impact of CIR margin for different cell loads and we have always found the same optimal CIR. These results are not presented in the paper. As we can see in figure 7, the optimal CIR margin is equal to the half of the power control step approximately. This can be justified by the fact that within the stable phase, the majority of user's CIRs oscillate around $\mathrm{CIR}_{\text {target }}^{M}$ with a standard deviation around $0.5 \mathrm{~dB}$. Therefore, the CIRs of these mobiles do not fall below $\mathrm{CIR}_{\text {target }}$ which is equivalent to $\mathrm{CIR}_{\text {target }}^{M}-0.5$ in this case. 

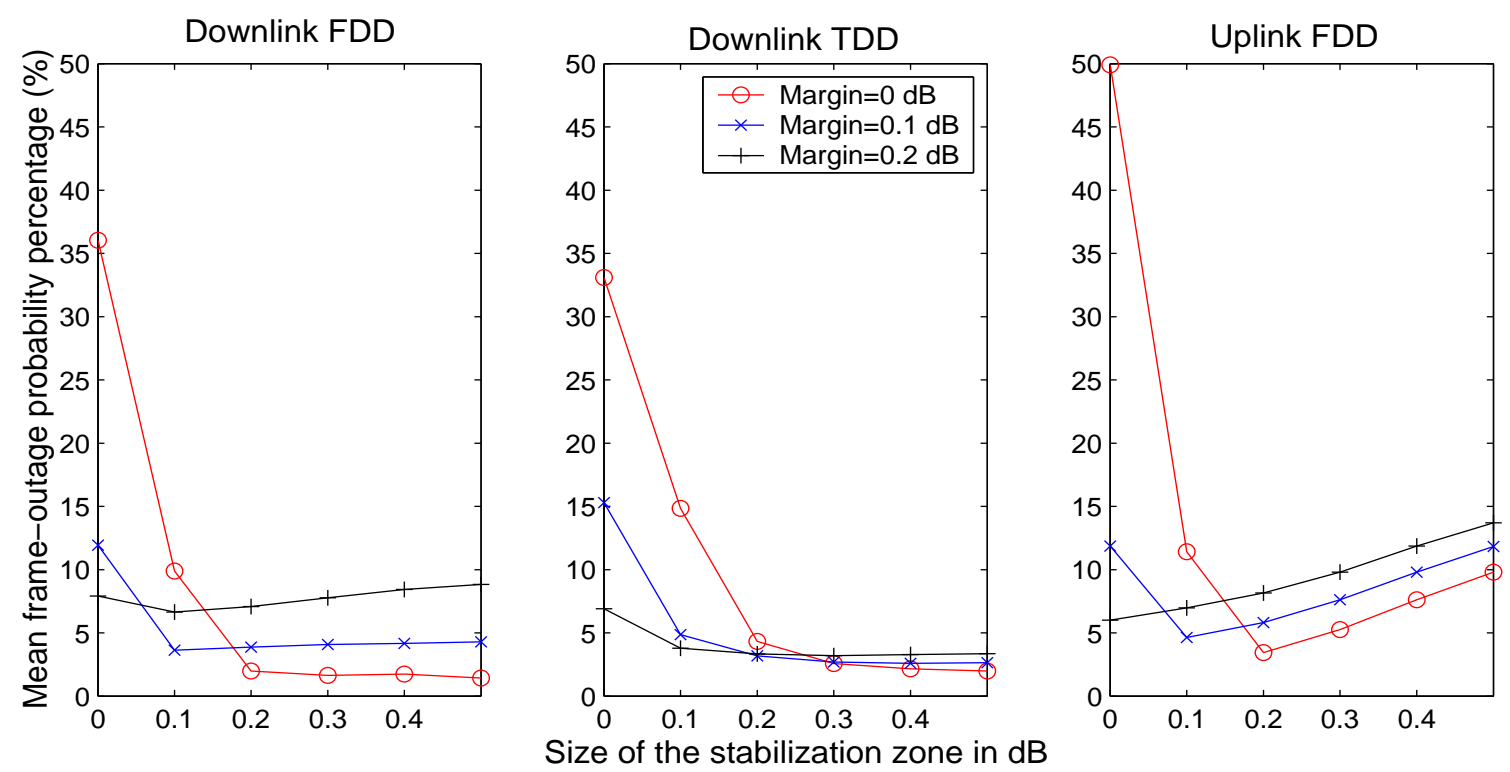

Figure 8: The mean frame-outage probability percentage as a function of stabilization zone size $\Phi$ for different values of the $C I R$ margin.

\subsection{Stabilization Zone Impact}

The impact of the stabilization zone size combined with a CIR margin on the MAPC algorithm is shown in figure 8 for the same loads as in figure 7 . This figure shows that the optimal value of $\Phi$ can be fixed to $0.3 \mathrm{~dB}$ with no CIR margin for the downlink and 0.2 for uplink. Besides, we notice that the stabilization zone size slightly changes the mean frame-outage probability when no CIR margin is used and $\Phi$ is greater than $0.3 \mathrm{~dB}$ especially in the downlink. This behavior makes the algorithm more feasible in systems where CIR measure sensitivity is not very high. By analyzing simulation results more closely, we have noticed the following remarks that explains the behavior of the proposed methods in the three cases:

$\diamond$ In downlink, the outage of mobiles is due to the remaining oscillation and power constraints are generally not attained. Therefore, outage probability decreases slightly when the value of $\Phi$ is higher than $0.3 \mathrm{~dB}$. The increase of stabilization zone size decreases the oscillation but in expense of an increase of power transmission. However, power transmission increase can be tolerated as the power constraint is not reached.

$\diamond$ In contrast with downlink, the outage of mobiles in uplink is due to power constraints (i.e. all mobiles in outage are transmitting with the maximum power). Therefore, an increase in the size of stabilization zone leads to an increase in the transmitted power; hence, the outage probability increases.

It should be noted that the capacity of the downlink is more sensitive to oscillation than in uplink due to the fact that the number of transmitters is very small in downlink. The small number of transmitters in downlink leads to high variations in intercell interference as a response for the variation in the transmitted power. In uplink, the high number of mobiles leads to the stabilization of the intercell interference around the mean value. 

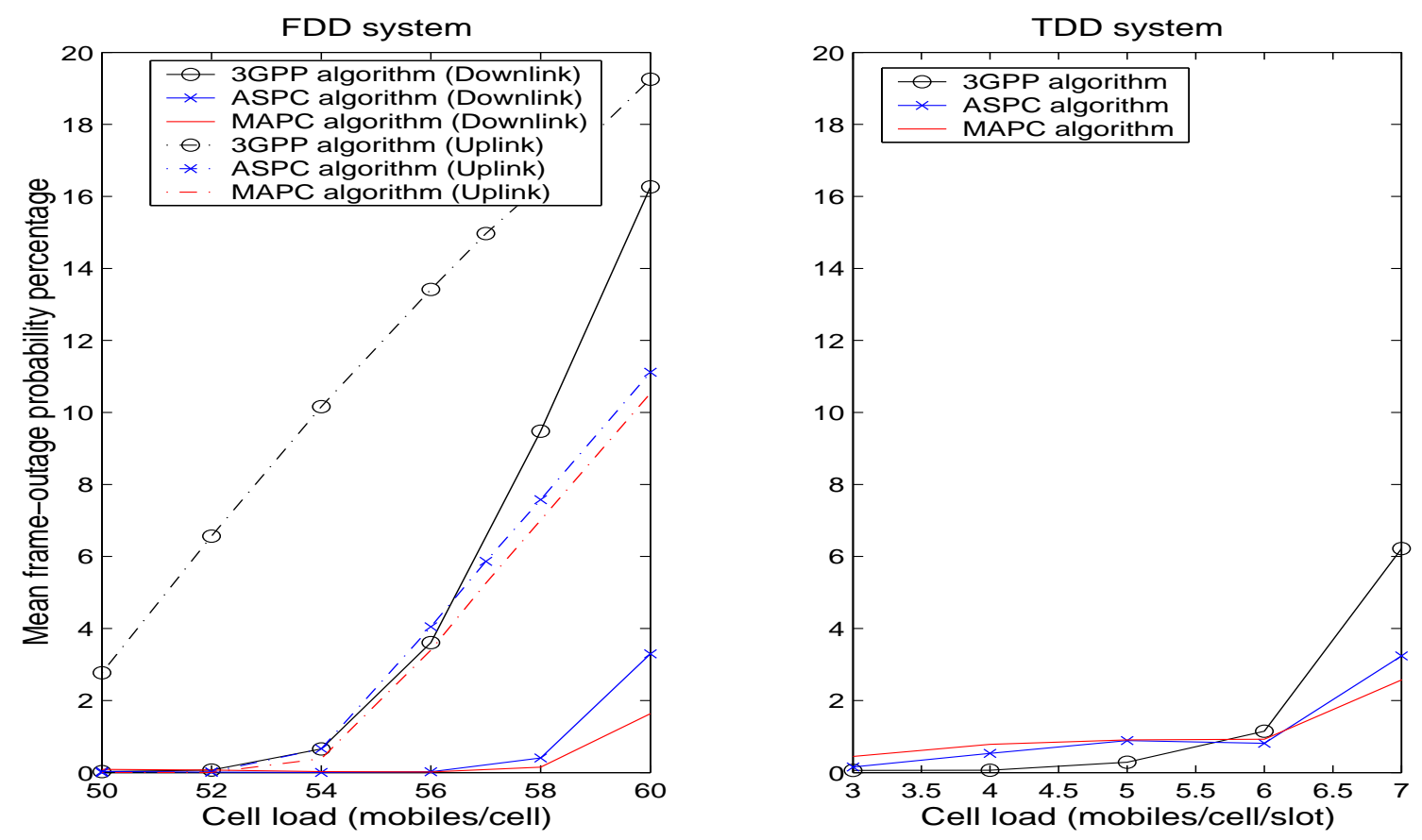

Figure 9: The mean frame-outage probability percentage for different cell loads and a fixed user configuration.

\subsection{Cell Load Impact}

In figure 9, the mean frame-outage probability percentage is plotted as a function of cell load. The plotted results are the best results obtained by the studied algorithms: the 3GPP algorithm with a $0.6 \mathrm{~dB}$ CIR margin, the ASPC with $0.2 \mathrm{~dB}$ margin and the MAPC algorithm with a stabilization zone of $0.3 \mathrm{~dB}$ in downlink and 0.2 in uplink.

The MAPC algorithm gives the lowest frame-outage probability. The difference in the mean frame-outage probability percentages between the MAPC algorithm and existing algorithms is an increasing function of cell load. This property makes the MAPC algorithm suited in systems with high traffic load.

It should be noted that the mean frame-outage probability is considered without error detection and correction; therefore, the capacity of a real system will be higher when these procedures are used.

In figure 10, the mean total transmitted power for downlink and mobile transmitted powers in uplink are plotted as a function of cell load. This figure shows that the MAPC algorithm reduces the total transmitted power of at least $1 \mathrm{~dB}$ from the ASPC algorithm for high loads and more than $3 \mathrm{~dB}$ from the $3 \mathrm{GPP}$ algorithm with the least outage probability. Moreover, mobiles transmitted powers in uplink are drastically reduced for low loads (e.g. $25 \mathrm{~dB}$ of reduction from the $3 \mathrm{GPP}$ algorithm and $4 \mathrm{~dB}$ from the ASPC algorithm in the FDD mode). Furthermore, the power increase in adaptive algorithm is a convex function of cell load, while it is a concave function in the $3 \mathrm{GPP}$ algorithm; therefore, the convergence of total transmitted powers to the maximum allowed power is slower in adaptive algorithms. In a dynamic system where pathgains are not fixed and where the instantaneous cell load is variable, the mean power consumption is thus, reduced by adaptive algorithms. 

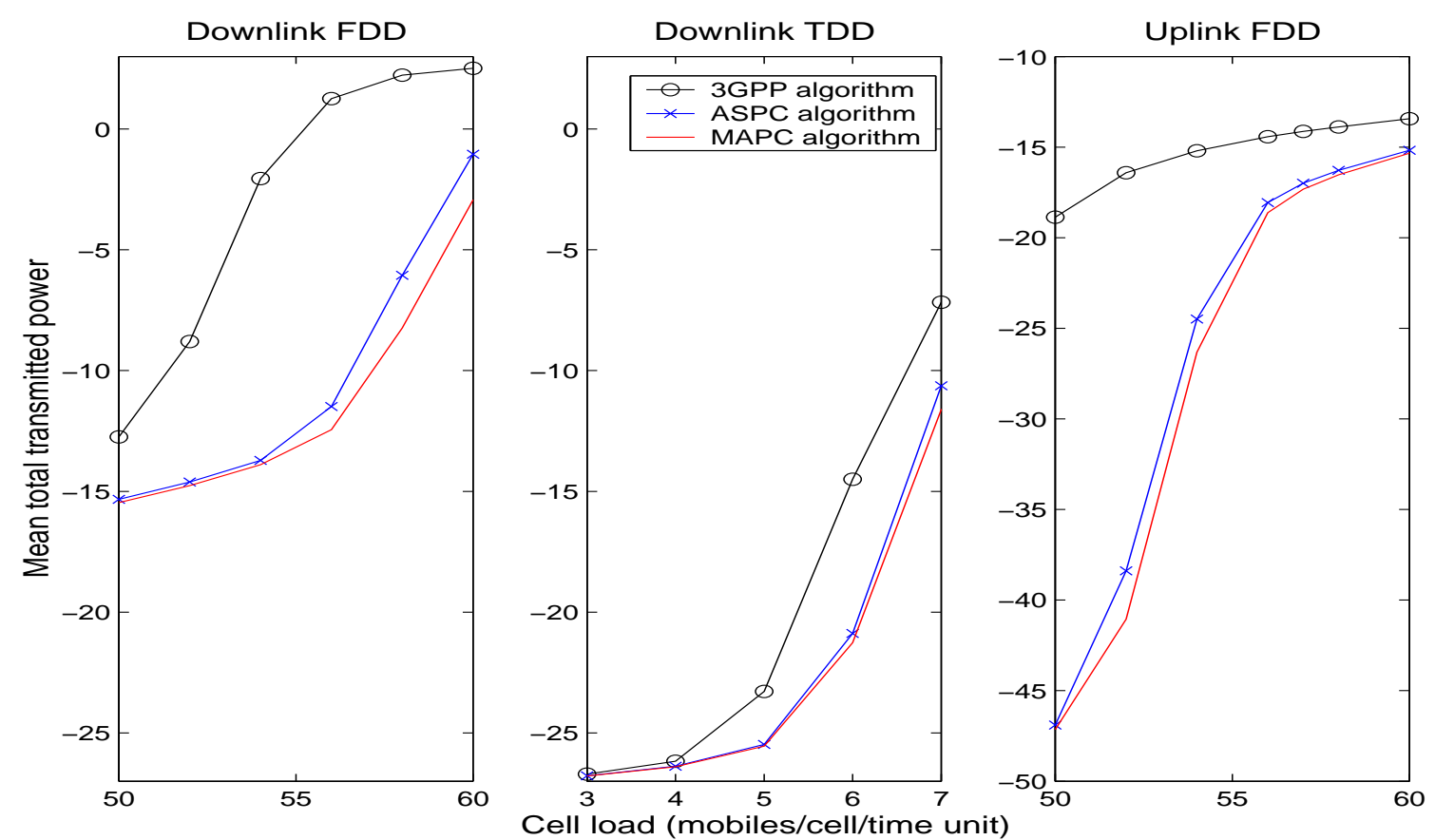

Figure 10: The mean total transmitted power as a function of the cell load.

\subsection{Mobility and Fading Impacts}

In this section, we only present the impact of user mobility and fading on the downlink of the FDD mode.

In figure 11, the cumulative distribution functions of the frame-outage probability are plotted for an averaged cell load of 60 pedestrian mobiles in each microcell with the same power control parameter as in the previous sections. This figure shows that the proposed algorithm decreases the frame-outage probability in a dynamic system with slow mobility due to the slow power increase in the adaptive algorithms as shown in the snapshot simulations. In figure 12 , we can notice that the MAPC algorithm decrease significantly the standard deviation of the SIR during one TTI (e.g. the mean value of the standard deviation given by the MPAC is decreased by $35 \%$ and $40 \%$ respectively from the mean values given by the ASPC and the $3 \mathrm{GPP}$ algorithms). Moreover, the standard deviation in the case of ASPC and MAPC algorithm has a higher variation range from the $3 \mathrm{GPP}$ algorithm due to the possibility of using high power steps. In figure 13, the cumulative distribution function of the total transmitted power is plotted. The latter figure shows that the MAPC algorithm reduces significantly the total transmitted power (e.g. the mean value is decreased by $0.5 \mathrm{~dB}$ and $1 \mathrm{~dB}$ when compared to the mean value given by the ASPC and the $3 \mathrm{GPP}$ algorithms respectively). It should be noted that the curves are similar to truncated lognormal distribution due to power constraints.

Moreover, we show in figures 14, 15 and 16 the results when the model with vehicular users is used. The results are obtained for a new set of power control parameters that optimize the performance of power control algorithms in a vehicular model (table II). In fact, when user's speed increases, conducting to an increase in fading variation, the adaptive power control is no more able to carry out with the same parameters as in the case with slow users. In other word, the reaction of the power control algorithm will be always slower than fading variations and therefore, high power control steps are no more suited for this case. Furthermore, the results show that the MAPC gives always the lowest frame-outage probabilities with a stabilization zone of $0.7 \mathrm{~dB}$ and maintains a low SIR deviation (i.e. close to the SIR deviation given by 


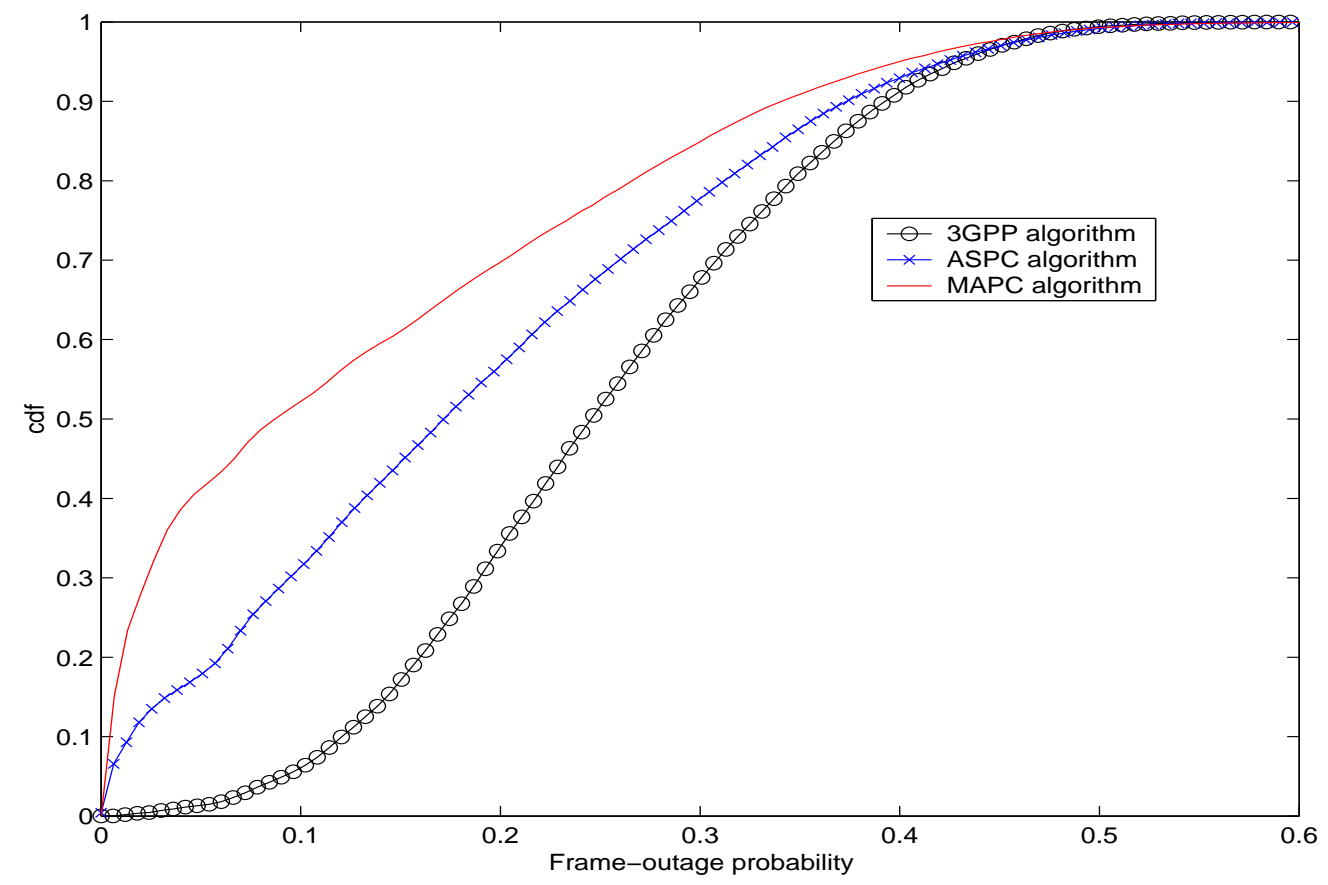

Figure 11: The frame-outage cumulative distribution function for a cell load of 60 mobiles/cell from dynamic simulations with pedestrian users.

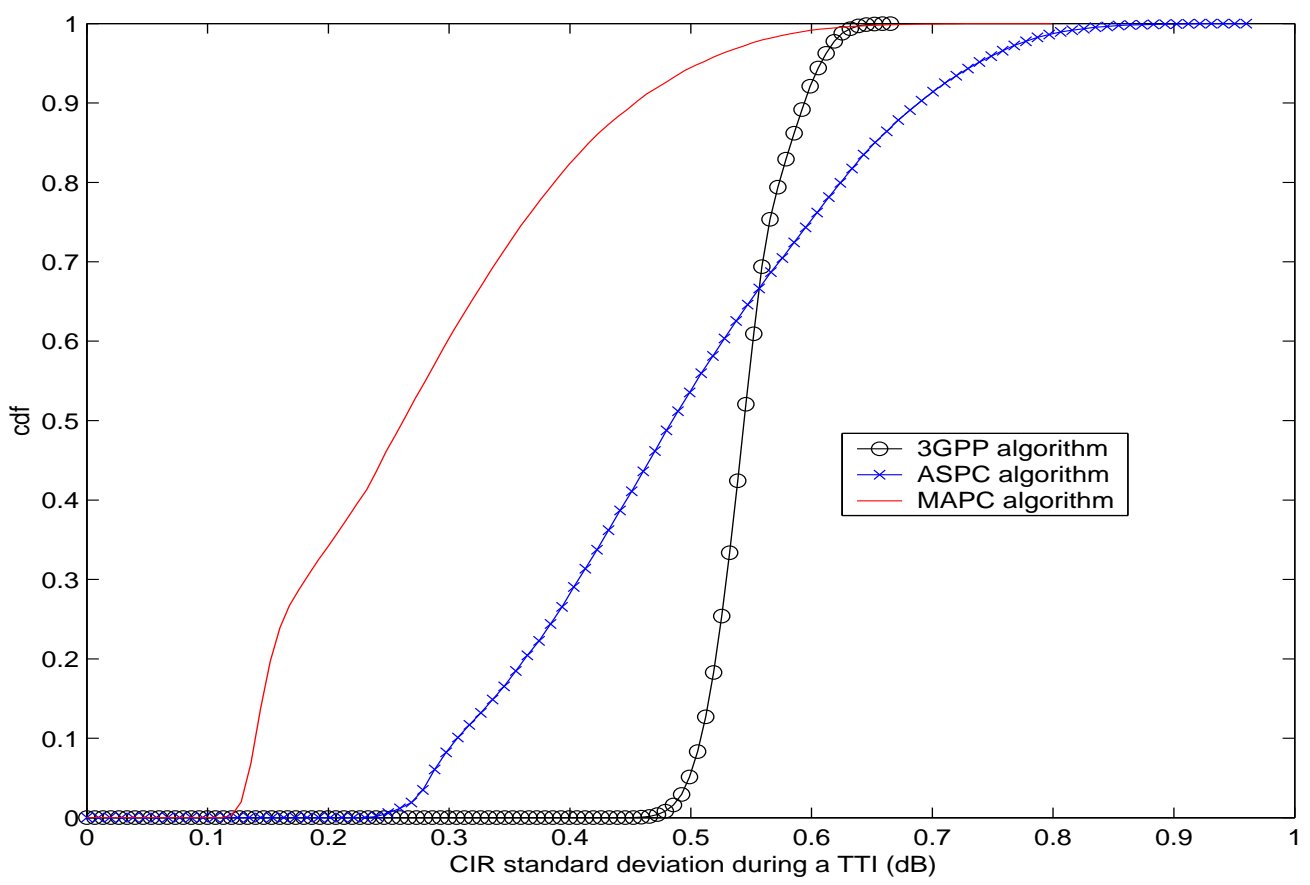

Figure 12: The cumulative distribution function of the $C I R$ standard deviation during one TTI for a cell load of 60 mobiles/cell from dynamic simulations with pedestrian users. 


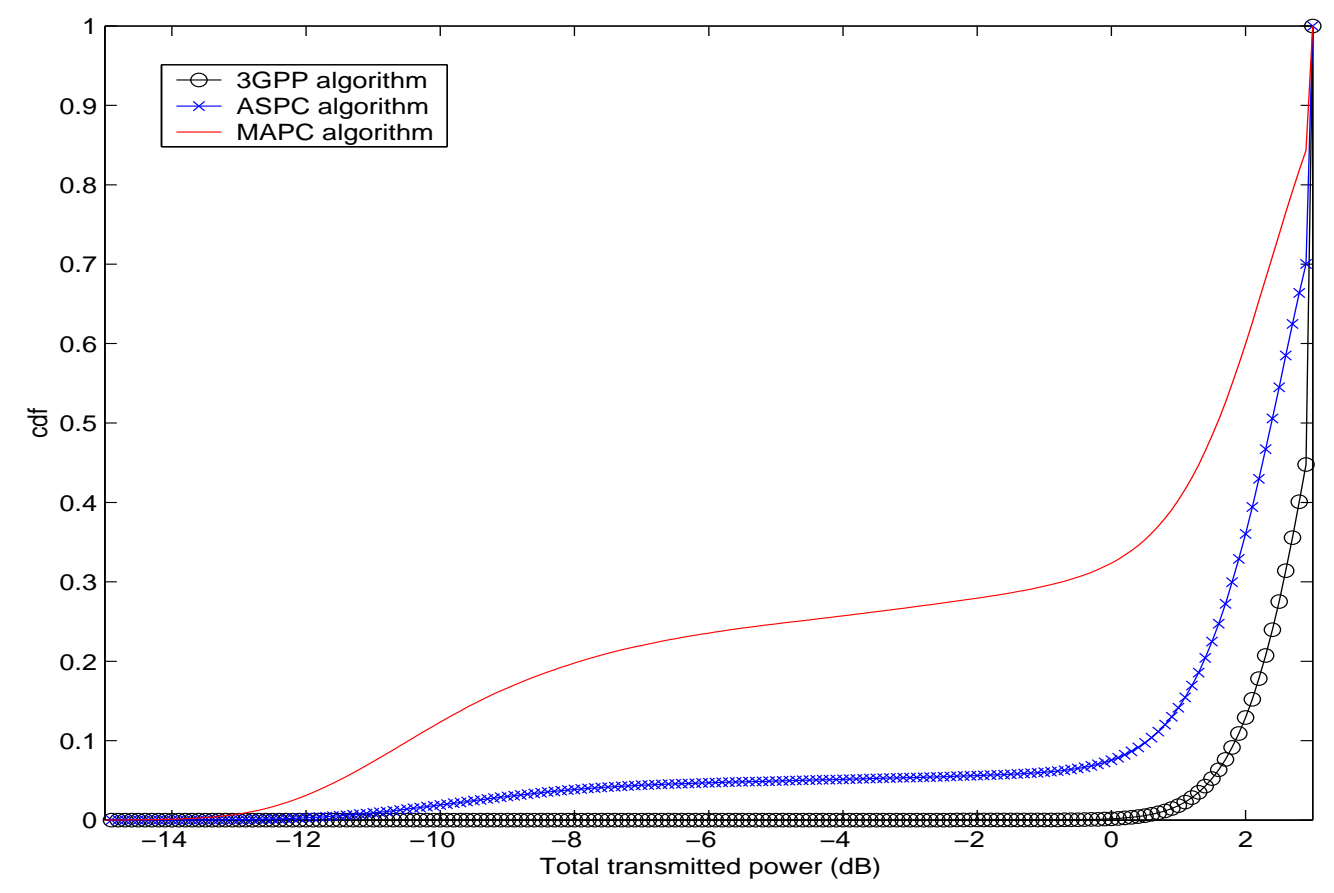

Figure 13: The cumulative distribution function of the total transmitted power of base stations for a cell load of 60 mobiles/cell from dynamic simulations with pedestrian users.

the 3GPP algorithm). Moreover, the stabilization zone size is increased in order to cope with the fast fading when power step values are limited. It should be noted also that the adaptive algorithms always have a power consumption lower than the 3GPP algorithm. In conclusion, these results show that the MAPC algorithm is advantageous in all type of systems thanks to its tunable parameters. These parameters can be fixed by the network planning tools for different type of user mobility.

\section{Concluding Remarks}

In this paper, we have presented the standardized power control for WCDMA systems. Thereafter, we have emphasized the fact that the standardized power control algorithm cannot always track channel fluctuations. Therefore, we have proposed a variant of the standardized algorithm that improves system performance in term of outage probability and power consumption. In order to limit oscillations, the modified adaptive power control algorithm forces the receiver to transmit an alternate sequence of up and down commands in a stabilization zone above the CIR target. The transmitter responds to this sequence by a power stabilization. Hence, only the interpretation of the TPC commands is ameliorated. Nevertheless, the proposed algorithm has shown better performance than the $3 \mathrm{GPP}$ and an existing adaptive algorithm; system maximum capacity is increased and the power consumption is reduced. This advantage is due to the elimination of the CIR margin. Moreover, the MAPC algorithm has shown good immunity to fast fading and user mobility if its parameters are carefully chosen.

A dynamic stabilization zone that depends on radio and services characteristics may be used for each mobile to increase the algorithm performance. This is left for future research. 


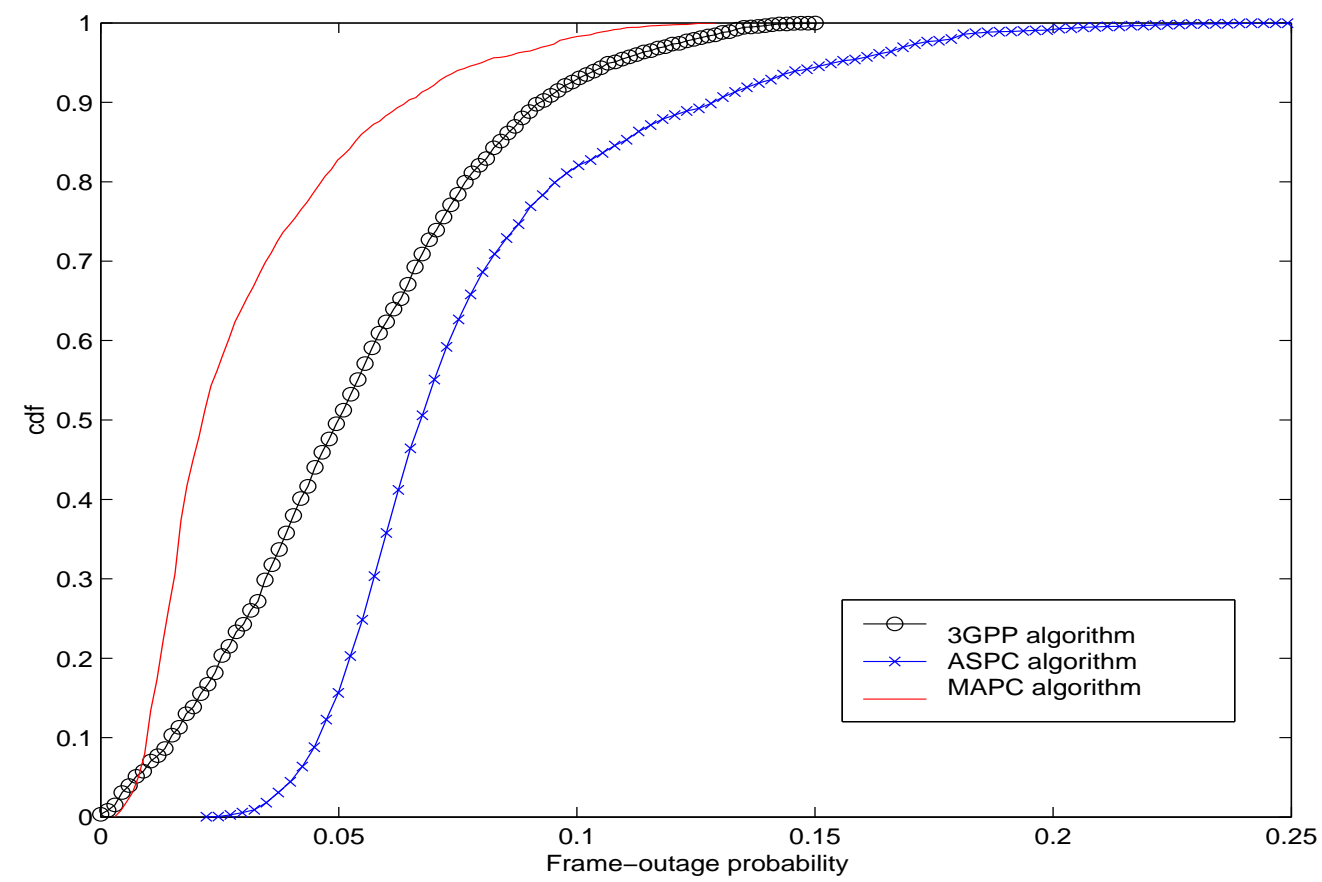

Figure 14: The frame-outage cumulative distribution function for a cell load of 40 mobiles/cell from dynamic simulations with vehicular users.

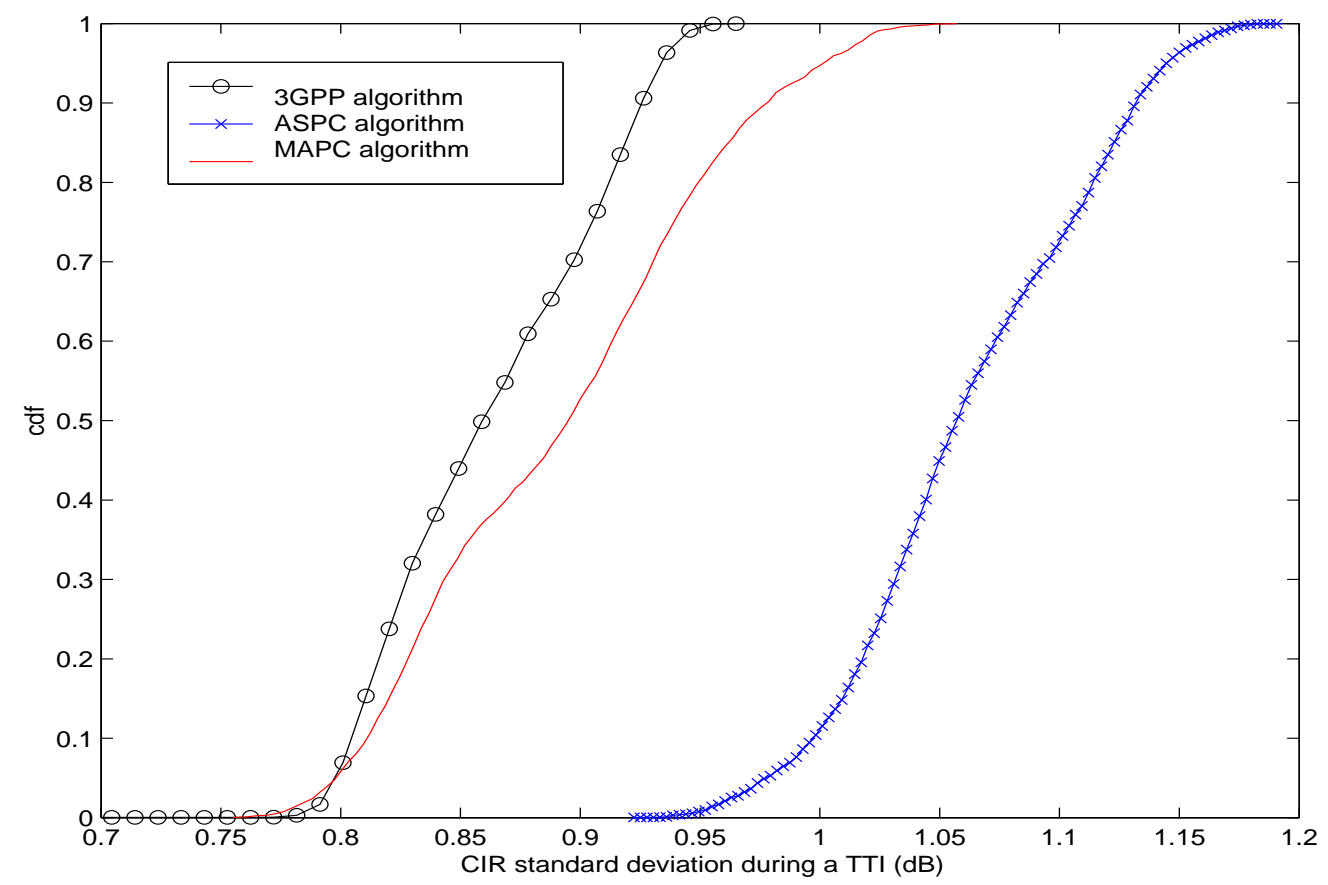

Figure 15: The cumulative distribution function of the $C I R$ standard deviation during one TTI for a cell load of 40 mobiles/cell from dynamic simulations with vehicular users. 


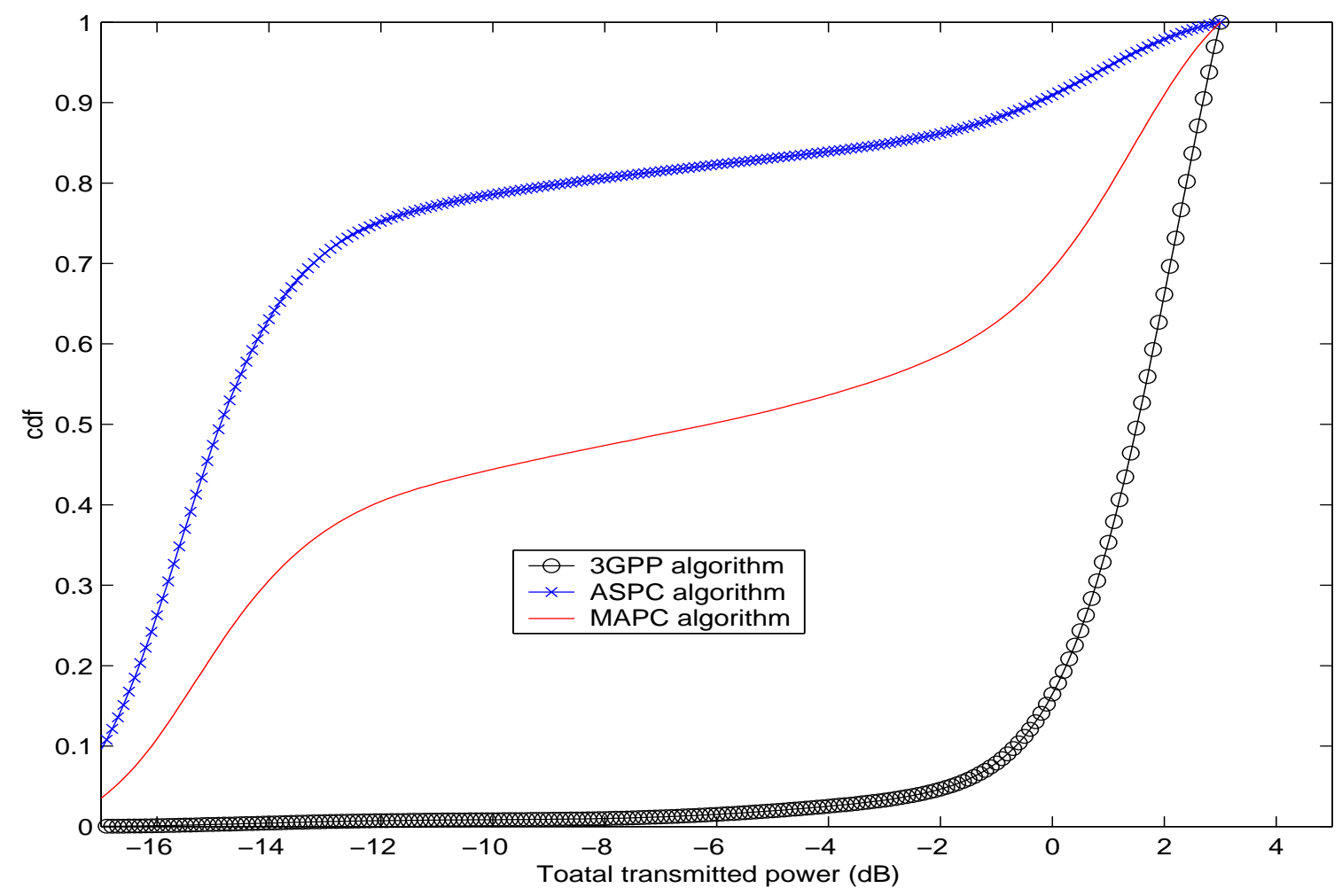

Figure 16: The cumulative distribution function of the total transmitted power of base stations for a cell load of 40 mobiles/cell from dynamic simulations with vehicular users.

\section{References}

[1] "Physical layer procedures (FDD)", Third Generation Partnership Project, Technical Specification Group Radio Access Network, TS25.214 (Release 5), September 2003.

[2] "Physical layer procedures (TDD)", Third Generation Partnership Project, Technical Specification Group Radio Access Network, TS25.224 (Release 5), September 2003.

[3] "(UTRAN) overall description", Third Generation Partnership Project, Technical Specification Group Radio Access Network, TS25.401 (Release 5), June 2003.

[4] Haardt M., Klein A., Koehn R., Oestreich S., Purat M., Sommer V., and Ulrich T., "The TD-CDMA based UTRA TDD mode", IEEE Journal on Selected Areas in Communications, vol. 18, pp. 1375 - 1385, 2000.

[5] Kurjenniemi J., Lehtinen O., and Ristaniemi T., "Improving utra tdd downlink power control with asymmetrical steps", in Proc.of the 57th IEEE Semiannual Vehicular Technology Conference (VTC Spring 2003), 2003, vol. 4, pp. $2480-2484$.

[6] Nuaymi L., Lagrange X., and Godlewski P., "A power control algorithm for 3G WCDMA system", in Proc. of the european Wireless conference, 2002.

[7] Naghian S., Rintamaki M., and Baghaie R., "Dynamic step-size power control in UMTS", in Proc. of The 13th IEEE International Symposium on Personal, Indoor and Mobile Radio Communications, 2002, vol. 4, pp. $1606-1610$. 
[8] Virtej I., Rintamaki M., and Koivo H., "Enhanced fast power control for WCDMA systems", in Proc.of The 11th IEEE International Symposium on Personal, Indoor and Mobile Radio Communications, 2000, pp. 1435 - 1439.

[9] Virtej I., Kansanen O., and Koivo H., "Enhanced predictive fast power control for 3G systems", in Proc.of The 53rd IEEE Vehicular Technology Conference (VTC 2001 Spring), 2001, vol. 4, pp. $2864-2868$.

[10] Rintamaki M., Virtej I., and Koivo H., "Two-mode fast power control for WCDMA systems", in Proc.of The 53rd IEEE Vehicular Technology Conference (VTC 2001 Spring), 2001, vol. 4, pp. $2893-2897$.

[11] Nasreddine J., Nuaymi L., and Lagrange X., "Adaptive power control algorithm for 3G cellular CDMA networks", in Proc.of the 59th IEEE Semiannual Vehicular Technology Conference (VTC04), 2004.

[12] "Universal mobile telecommunications system (UMTS); selection procedures for the choice of radio transmission technologies of the UMTS", TR 101 112 (UMTS 30.03), April 1998.

[13] Gudmundson M., "Correlation model for shadow fading in mobile radio systems", Electronics Letters, vol. 27, pp. 2145-2146, 1991.

[14] Jakes W.C., Microwave Mobile Communications, New York: John willey sons, 1974.

[15] "Deployment aspects", Third Generation Partnership Project, Technical Specification Group Radio Access Network, TS25.943 (Release 5), June 2002.

[16] Ammari S. and Wautier A., "Interference factor evaluation for CDMA system capacity analysis", in the 53rd IEEE Vehicular Technology Conference (VTC Spring 2001), 2001, vol. 3, pp. $1573-1577$.

[17] "RF system scenarios", Third Generation Partnership Project, Technical Specification Group Radio Access Network, TS25.942 (Release 5), June 2002.

[18] Holma H., A Study of UMTS terrestrial radio access performance, Helsinki University of Technology Communications Laboratory Technical Report T49, 2003. 